\title{
COMMON FIXED POINTS OF COMMUTING HOLOMORPHIC MAPPINGS
}

\author{
By T. Kuczumow and A. STAchura
}

\begin{abstract}
Let $B_{k}$ be a unit open ball in a $k$-dimensional complex Hilbert space. If $T_{\mu}$ is a commuting family of continuous functions mapping $B_{k}^{n}$ into itself and holomorphic in $B_{k}^{n}$, then there exists a common fixed point for all functions of this family.
\end{abstract}

Introduction. Let us consider a family $\mathcal{A}$ of mappings of some set into itself. If $T x=x$ for all $T$ in $\mathcal{A}$ and some $x$ we say that $x$ is a common fixed point for $\mathcal{A}$ (or for the mapping $T$ in $\mathcal{A}$ ). In this paper we are concerned with the existence of common fixed points for families of mappings.

For many years it was unknown whether two commuting continuous mappings of a compact convex set into itself necessarily have a common fixed point. In 1969 Boyce ([2]) and Huneke ([13]) independently gave counterexamples: there exist two commuting continuous mappings of $[0,1]$ into itself without a common fixed point. In view of this it is not surprising that the positive results must involve some additional restrictions on the family $A$. Throughout this paper $A$ denotes a subfamily of a family of all mappings from $\bar{B}^{n}$ into $\bar{B}^{n}$ ( $B^{n}$ is a cartesian product of $n$ open unit balls $B$ of a Hilbert space $H$ ). The mappings in $\mathcal{A}$ are holomorphic on $B^{n}$ and continuous on $\bar{B}^{n}$.

In [21] (see also [3] and [4]) Shields proved that if $A$ is a family of commuting functions which are continuous on the closed disc $\bar{\Delta}$ of the complex plane and are holomorphic on the open disc $\Delta$ and map the closed disc into itself, then there exists a common fixed point for all the functions of the family A. This result was extended to polydiscs in $\boldsymbol{C}^{2}$ by Eustice ([6]) (see also [24]) and to the unit ball of a finite dimensional inner product space by Suffridge ([23]). In [11] Heath and Suffridge gave the following theorem. If $T_{1}$ and $T_{2}$ are continuous mappings of a polydisc $\bar{\Delta}^{n}$ into itself that are holomorphic on $\Delta^{n}$ and $T_{1} \circ T_{2}=T_{2} \circ T_{1}$, then they have a common fixed point in $\bar{\Delta}^{n}$. However we think that the proof in [11] is not complete.

In each mentioned above paper the proof is based on the fact that if a closure of the iterates of $T$ (denoted by $\Gamma(T)$ ) is a compact topological semigroup then it contains a unique idempotent. Because of this our problem reduces

Received September 19, 1988; Revised March 2, 1989. 
to a study of idempotents in $\mathcal{A}$. The absolutely different methods allow us to prove the following two facts. If $B$ is an open unit ball of a Hilbert space and $\mathcal{A}$ is a commuting family of continuous functions mapping $\bar{B}$ into itself and holomorphic in $B$, then there exists a common fixed point for $\mathcal{A}$ ([14], [17]) and if $T_{1}, \cdots, T_{m}: B^{n}-B^{n}$ are commuting and holomorphic and every mapping has a fixed point, then they have a common fixed point ([16], [17]).

Basic notations and facts. We shall use the following notations:

(i) $H\left(H_{k}\right)$ is a complex Hilbert space (a complex Hilbert space with $\operatorname{dim} H_{k}$ $=k$ ) with an inner product $(\cdot, \cdot)$ and a norm $\|\cdot\|$.

(ii) $B\left(B_{k}\right)$ is a unit open ball in $H\left(\right.$ in $\left.H_{k}\right)$ and $\bar{B}\left(\bar{B}_{k}\right)$ is a closure of $B$ $\left(B_{k}\right)$ in $H\left(H_{k}\right)$.

(iii) In $B^{n}$ we introduce the following CRF metric $\rho_{n}$ ([7], [8], [9], [10], [26]):

$$
\rho_{1}(x, y)=\tanh ^{-1}\left(1-\frac{\left(1-\|x\|^{2}\right)\left(1-\|y\|^{2}\right)}{|1-(x, y)|^{2}}\right)^{1 / 2}
$$

for $x, y \in B^{1}=B$ and

$$
\rho_{n}\left(\left(x_{1}, \cdots, x_{n}\right),\left(y_{1}, \cdots, y_{n}\right)\right)=\max _{1 \leqq \imath \leqq n} \rho_{1}\left(x_{\imath}, y_{\imath}\right)
$$

for $\left(x_{1}, \cdots, x_{n}\right),\left(y_{1}, \cdots, y_{n}\right) \in B^{n}$.

(iv) $\mathscr{H}\left(B^{n}\right)\left(\mathscr{H}\left(B_{k}^{n}\right)\right)$ is the set of holomorphic mappings of $B^{n}$ into $B^{n}$ (of $B_{k}^{n}$ into $\left.B_{k}^{n}\right) . \mathscr{H}\left(B_{k}^{n}, H_{k}^{n}\right)$ is the set of holomorphic mappings of $B_{k}^{n}$ into $H_{k}^{n}$.

(v) For $T \in \mathscr{H}\left(B_{k}^{n}\right) \Gamma(T)$ denotes the closure in $\mathscr{T}\left(B_{k}^{n}, H_{k}^{n}\right)$ of the iterates of $T$ in the topology of the uniform convergence on compact subsets of $B_{k}^{n}$. $\Gamma(T)$ is a compact set in this topology.

(vi) For $T \in \mathscr{H}\left(B_{k}^{n}\right) \Gamma^{\prime}(T)$ denotes the set $\left(\subset \mathscr{H}\left(B_{k}^{n}, H_{k}^{n}\right)\right)$ of all subsequential limits of $\left\{T^{p}\right\}$ in the topology of the uniform convergence on compact subsets of $B_{k}^{n}$.

If $\Gamma(T) \subset \mathscr{H}\left(B_{k}^{n}\right)$, then it forms a compact topological semigroup. Let us notice that this topological semigroup $\Gamma(T)$ contains exactly one idempotent $R_{T}$ ([12], [28]). This holomorphic idempotent in $\Gamma(T)$ is a holomorphic retraction of $B_{k}^{n}$. In [1], [6], [11], [16], [23] and [27] it is shown what such a retraction looks like.

Every holomorphic mapping $T \in \mathscr{H}\left(B^{n}\right)$ is nonexpansive in $\left(B^{n}, \rho_{n}\right)$ and has a fixed point if and only if there exists $x \in B^{n}$ such that a sequence of its iterates $\left\{T^{p} x\right\}$ is bounded in $\left(B^{n}, \rho_{n}\right)([8],[26])$.

Common fixed points. First we require the following results concerning $\Gamma(T)$ and $\Gamma^{\prime}(T)$.

THEOREM 1. Let $T: B_{k}^{n} \rightarrow B_{k}^{n}$ be a holomorphic mapping. The following statements are equivalent: 
(i) T has a fixed point,

(ii) $\Gamma(T) \subset \mathscr{H}\left(B_{k}^{n}\right)$,

(iii) $\Gamma(T)$ contains a holomorphic retraction $R_{T} \in \mathcal{H}\left(B_{k}^{n}\right)$,

(iv) $\Gamma^{\prime}(T)$ contains $F \in \mathscr{H}\left(B_{k}^{n}\right)$,

(v) there exist $x_{0}$ and $\left\{p_{i}\right\}$ such that $\sup _{i}\left\|T^{p_{i}}\left(x_{0}\right)\right\|<1$.

Proof.

(i) $\Rightarrow$ (ii). If $T$ has a fixed point, then for every $x \in B_{k}^{n}$ the sequence $\left\{T^{p} x\right\}$ is $\rho_{n}$-bounded and it gives $\Gamma(T) \subset \mathcal{H}\left(B_{k}^{n}\right)$.

(ii) $\Rightarrow$ (iii). If $\Gamma(T) \subset \mathcal{H}\left(B_{k}^{n}\right)$ then $\Gamma(T)$ is a compact abelian semigroup and therefore $\Gamma(T)$ contains a holomorphic idempotent which is a holomorphic retraction.

(iii) $\Rightarrow$ (iv). Obvious.

(iv) $\Rightarrow$ (v). Obvious.

$(\mathrm{v}) \Rightarrow(\mathrm{i})$. If for $x_{0} \in B_{k}^{n}$ there exists $\left\{p_{i}\right\}$ such that $\sup _{i}\left\|T^{p_{i}}\left(x_{0}\right)\right\|<1$ then the sequence $\left\{T^{p_{i}}\left(x_{0}\right)\right\}$ is $\rho_{n}$-bounded. By the theorem of Całka ([5]) this subsequence $\left\{T^{p_{i}}\left(x_{0}\right)\right\}$ of $\left\{T^{p}\left(x_{0}\right)\right\}$ guarantees the $\rho_{n}$-boundedness of $\left\{T^{p}\left(x_{0}\right)\right\}$ and hence $T$ has a fixed point.

Remark 1. It is worth noticing that if $B \subset H$ and $\operatorname{dim} H=+\infty$ the implication $(v) \Rightarrow(i)$ is not true $([22])$.

Directly from the above proof we get

THEOREM 2. Let $T: B_{k}^{n} \rightarrow B_{k}^{n}$ be a holomorphic mapping. $T$ is fixed point free if and only if for every $F \in \Gamma^{\prime}(T)$ the image $F\left(B_{k}^{n}\right)$ is contained in the boundary of $B_{k}^{n}$.

Theorem 1 allows us to prove the main theorem about the existence of common fixed points.

THEOREM 3. Let $\left\{T_{\mu}\right\}$ be a commuting family of continuous functions mapping $\bar{B}_{k}^{n}$ into itself and holomorphic in $B_{k}^{n}$. Then there is a common fixed point for all functions of the family.

Proof. It is sufficient to prove this theorem for a finite family $\left\{T_{1}, \cdots, T_{m}\right\}$.

Case 1. Every $T_{i \mid B_{k}^{n}}$ has a fixed point in $B_{k}^{n}$. Here the existence of a common fixed points is proved in [14] and [15]. For the reader's convenience we give a sketch of the proof of this fact.

Every Fix $\left(T_{i \mid B_{k}^{n}}\right)$ is a holomorphic retract of $B_{k}^{n}$ ([16], [27]). "- Since $\left\{T_{1}\right.$, $\left.\cdots, T_{m}\right\}$ is a commuting family of functions we have

$$
T_{j}\left(\operatorname{Fix}\left(T_{i \mid B_{k}^{n}}^{n}\right)\right) \subset \mathrm{Fix}\left(T_{i \mid B_{k}^{n}}\right)
$$

for $1 \leqq i, j \leqq m$. 
Next if $R: B_{k}^{n}-A \subset B_{k}^{n}$ is a holomorphic retraction and $T: B_{k}^{n}-B_{k}^{n}$ is a holomorphic mapping with $\operatorname{Fix}(T) \neq \varnothing$ and $T(A) \subset A$, then $A \cap \operatorname{Fix}(T)$ is a nonempty holomorphic retract of $B_{k}^{n}$. Indeed $T \circ R$ has a fixed point (see (v) in Theorem 1) and every such a point lies in $A$. It is easy to observe that $\operatorname{Fix}(T \circ R)=$ $A \cap \operatorname{Fix}(T)$.

Now it is sufficient to apply a mathematical induction with respect to $m$ to obtain a common fixed point of $\left\{T_{1}, \cdots, T_{m}\right\}$.

In the next two cases we proceed by induction with respect to $n$. For $n=1$ see [14] and [23].

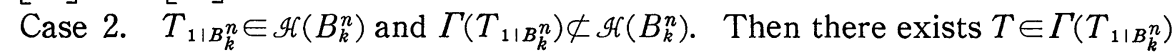
such that (after applying of an appropriate linear mapping $L$ that permutes coordinates)

$$
T\left(B_{k}^{n}\right) \subset\left\{e_{1}\right\} \times \cdots \times\left\{e_{q}\right\} \times B_{k}^{n-q}
$$

$(1 \leqq q \leqq n)$ and $T, T_{1}, \cdots, T_{m}$ commute. It is clear that

$$
T_{i}\left(\left\{e_{1}\right\} \times \cdots \times\left\{e_{q}\right\} \times B_{k}^{n-q}\right) \subset\left\{e_{1}\right\} \times \cdots \times\left\{e_{q}\right\} \times \bar{B}_{k}^{n-q}
$$

$(1 \leqq \imath \leqq m)$ and therefore $T_{1}, \cdots, T_{m}$ have a common fixed point in $\left\{e_{1}\right\} \times \cdots$ $\times\left\{e_{q}\right\} \times \bar{B}_{k}^{n-q}$ by the induction hypothesis.

Case 3. $T_{1 \mid B_{k}^{n}} \notin \mathscr{H}\left(B_{k}^{n}\right)$. By the induction hypothesis $T_{1}, \cdots, T_{m}$ have a common fixed point (see Case 2).

Remark 2. A continuous mapping $T: \bar{B}^{n} \rightarrow \bar{B}^{n}$ which satisfies the following condition

$$
\rho_{n}(t T x, t T y) \leqq \rho_{n}(x, y)
$$

for all $x, y \in B^{n}$ and every $0 \leqq t<1$ is called a nonexpansive mapping in $\bar{B}^{n}$. Theorem 1 and 2 are still true if we replace the assumption of holomorphy of mappings in $B_{k}^{n}$ by nonexpansiveness in $\bar{B}_{k}^{n}$.

Open problem. If we have a mapping $T: \bar{B}^{n} \rightarrow \bar{B}^{n}$ which is nonexpansive in $\bar{B}^{n}$, then $T$ has a fixed point ([8], [9], [10], [15], [19], [20]). Is Theorem 2 true if we replace $\bar{B}_{k}^{n}$ by $\bar{B}^{n}$ ? If either $n=1$ or every $T_{\mu}$ is a $\rho_{n}$-isometry it is known that the answer is positive ([14], [17], [18]).

\section{REFERENCES}

[1] M. AbD-Alla, Sur l'ensemble des points fixes d'une application holomorphe, C.R. Acad. Sci. Paris Sér. I Math. 302 (1986), 451-454.

[2] W.M. Boyce, Commuting functions with no common fixed point, Trans. Amer. Math. Soc. 137 (1969), 77-92.

[3] R.B. BurCKel, Iterating analytic self-maps of disc, Amer. Math. Monthly 88 (1981), 396-407.

[4] R. B. BURCKel, An introduction to classical complex analysis, Vol. I., Birkhäuser 
Verlag, Basel and Stuttgart, 1979.

[5] A. CAŁKA, On conditions under which isometries have bounded orbits, Colloq. Math. XLVIII (1984), 219-227.

[6] D.J. Eustice, Holomorphic idempotents and common fixed points on the 2-disk, Michigan Math. J. 19 (1972), 347-352.

[7] T. FRANZONI AND E. VESENTINI, Holomorphic maps and invariant distances, North Holland, Amsterdam 1980.

[8] K. Goebel, Uniform convexity of Carathéodory's metric on the Hilbert ball and its consequences, Institute Nazionale di Alta Matematica Francesco Severi, Symposia Mathematica XXVI (1982), 163-179.

[9] K. Goebel and S. Reich, Uniform convexity, hyperbolic geometry and nonexpansive mappings, Marcel Dekker, New York and Basel, 1984.

[10] K. Goebel, T. Sekowski and A. Stachura, Uniform convexity of hyperbolic metric and fixed points of holomorphic mappings in the Hilbert ball, Nonlinear Analysis 4 (1980), 1011-1021.

[11] L.F. Heath AND T.J. Suffridge, Holomorphic retracts in complex $n$-space, Illinois J. Math. 25 (1981), 125-135.

[12] E. Hewitt and K. A. Ross, Abstract harmonic analysis. Vol. I, Academic Press, New York, 1963.

[13] J.P. Huneke, On common fixed points of commuting continuous functions on an interval, Trans. Amer. Math. Soc. 139 (1969), 371-381.

[14] T. Kuczumow, Common fixed points of commuting holomorphic mappings in Hilbert ball and polydisc, Nonlinear Analysis 8 (1984), 417-419.

[15] T. Kuczumow, Nonexpansive retracts and fixed points of nonepansive mappings in the cartesian product of $n$ Hilbert balls, Nonlinear Analysis 9 (1985), 601-604.

[16] T. Kuczumow, Holomorphic retracts of polyballs, Proc. Amer. Math. Soc. 98 (1986), 374-375.

[17] T. Kuczumow, Nonexpansive mappings and isometries of Hilbert $n$-balls with hyperbolic metrics, Habilitation Dissertation, UMCS Lublin, 1987.

[18] T. Kuczumow AND W.O. RaY, Isometries in the cartesian product of $n$ unit open Hilbert balls with a hyperbolis metric, Ann. Mat. Pura Appl. (to appear.).

[19] T. Kuczumow And A. Stachura, Convexity and fixed points of holomorphic mappings in Hilbert ball and polydisc, Bull. Polish Acad. Sci. Math. 34 (1986), 189-193.

[20] T. Kuczumow And A. Stachura, Fixed points of holomorphic mappings in the cartesian product of $n$ unit Hilbert balls, Canad. Math. Bull. 29 (1986), 281-286.

[21] A.L. SHIELdS, On fixed points of commuting analytic functions, Proc. Amer. Math. Soc. 15 (1964), 703-706.

[22] A. STACHURA, Iterates of holomorphic self-maps of the unit ball in Hilbert space, Proc. Amer. Math. Soc. 93 (1985), 88-90.

[23] T. J. Suffridge, Common fixed points of commuting holomorphic maps of the hyperball, Michigan Math. J. 21 (1974), 309-314.

[24] M. Suzuki, The fixed point set and the iterational limits of a holomorphic selfmaps, Kodai Math. J. 10 (1987), 298-306.

[25] E. Vesentini, Complex geodesics and holomorphic maps, Instituto Nazionale di Alta Matematica Francesco Severi, Symposia Mathematica XXVI (1982), 211-230.

[26] J.-P. ViguE, Points fixes d'applications holomorphes dans un produit fini de boulesunités d'espaces de Hilbert, Ann. Mat. Pura Appl. CXXXVII (1984), 245-256. 
[27] J.-P. VIGUE, Points fixes d'applications holomorphes dans un domaine borné convexe de $\boldsymbol{C}^{n}$, Trans. Amer. Math. Soc. 289 (1985), 345-353.

[28] A.D. WAllace, The structure of topological semigroups, Bull. Amer. Math. Soc. 61 (1955), 95-112.

INSTytut MATEMATyKi UMCS

Plac Maril Curie-Skłodowskiej 1

20-031 Lublin, Poland 